\title{
Driving and sorting of the fluorescent droplets on digital microfluidic platform
}

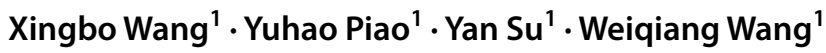

Received: 8 July 2018 / Accepted: 24 October 2018 / Published online: 1 November 2018

(c) The Author(s) 2018

\begin{abstract}
In this paper, we present a digital microfluidic droplet sorting platform to achieve automated droplet sorting based on fluorescent detection. We design and fabricate a kind of digital microfluidic chip for manipulating nano-liter-sized liquid droplets, and the chip is integrated with a fluorescence-initiated feedback system for real-time sorting control. The driving and sorting characteristics of fluorescent droplets encapsulating fluorescent-labeled particles are studied on this platform. The droplets dispensed from on-chip reservoir electrode are transported to a fluorescence detection site and sorted according to their fluorescence signals. The fluorescent droplets and non-fluorescent droplets are successfully separated and the number of fluorescent particles inside each droplet is quantified by its fluorescent intensity. We realize droplet sorting at $20 \mathrm{~Hz}$ and obtain a linear relationship between the fluorescent particle concentrations and the fluorescence signals. This work is easily adapted for sorting out fluorescent-labeled microparticles, cells and bacteria and thus has the potential of quantifying catalytic or regulatory bio-activities.
\end{abstract}

Keywords Droplet sorting $\cdot$ Digital microfluidics $\cdot$ Fluorescent particles $\cdot$ Fluorescent detection

\section{Introduction}

Fluorescent droplet sorting has great research value in biomedicine study and biology researches. Most of the droplet sorting methods are based on microfluidic channels using sheath fluid (Johansson et al. 2009; Mazutis et al. 2013; Xi et al. 2017) or syringe pump (Wu et al. 2013; Hung et al. 2015; Obexer et al. 2016). Among them, various triggered mechanisms have been demonstrated to achieve effective droplet sorting. For example, a kind of optical tweezers is reported to realize the sorting process (Eriksson et al. 2010) by gradient and scattering force to capture cells. A solenoid valve along with compressed air is used to create a bubble in an actuation channel to sort droplets filled with fluorescent

This article is part of the topical collection "2018 International Conference of Microfluidics, Nanofluidics and Lab-on-a-Chip, Beijing, China" guest edited by Guoqing Hu, Ting Si and Zhaomiao Liu.

Weiqiang Wang

wangweiqiang@njust.edu.cn

1 School of Mechanical Engineering, Nanjing University of Science and Technology, Nanjing 210094, Jiangsu, People's Republic of China beads (Cao et al. 2012). A laser pulse is also utilized to induce a cavitation bubble whose expansion produces a high-speed liquid jet that deflects the desired sample towards the collection channel for sorting (Chen et al. 2013). A fluorescence-activated sorting mechanism is reported to isolate droplets containing cells by hydrodynamic gated injection with side-channel stopped flow technique (Wu et al. 2013). Moreover, thermoreversible gelation polymers (Okada et al. 2014), piezoelectric activators (Zhao et al. 2014), surface acoustic wave actuations (Schmid et al. 2014) and dielectrophoresis actuations (Baret et al. 2009; Song et al. 2015; Qiao et al. 2017; Clark et al. 2018) have also been used to achieve fluorescent droplet separation.

These microfluidic systems have been established as valuable tools for droplet sorting. However, channel-based microfluidics are limited by the fact that particles might adhere to the walls of channel to cause blocking and failure of device. In addition, it is difficult to isolate and separate a specific droplet inside fluid channels. On the other hand, digital microfluidic (DMF) manipulates individual droplets by electric actuation and thus has the advantage of maximum flexibility and natural labeling of each droplet. In digital microfluidics, it is not only easy to locate and separate a target droplet, but it is also capable of realizing complex fluid 
operations. For example, some biological assays require washing steps, in which reagents from a first reaction must be completely removed before a new set of reagents is introduced. Such heterogeneous assays are difficult to perform inside fluidic channels, but a washing step can be easily done on digital microfluidic chips (Hung et al. 2017).

In this paper, we study the driving and sorting characteristics of nano-liter-sized fluorescent droplets on the digital microfluidic platform. Through a real-time fluorescent intensity measurement and feedback sorting control, the droplets containing fluorescent particles are successfully separated from those without fluorescent particles and the fluorescent particle concentration is determined by the fluorescent intensity.

\section{DMF chip design and fabrication}

The DMF chip based on the principle of electrowettingon-dielectric is composed of two parallel plates with liquid droplets sandwiched in between. The DMF chip is fabricated on a transparent glass substrate and the layout of bottom electrodes is shown in Fig. 1. The inverted T-shaped electrode structure for manipulating droplets includes reservoir electrode, dispensing electrode, transport electrodes, detection site, and collecting electrodes. At first the solution to be sorted is placed on the reservoir electrode. Then droplets are generated from the reservoir by dispensing progress. Subsequently, the dispensed droplets are transported to the detection site where a transparent circle was designed for fluorescence measurement. After passing the detection site, the droplets are sorted according to its fluorescent intensity and transported to different directions.

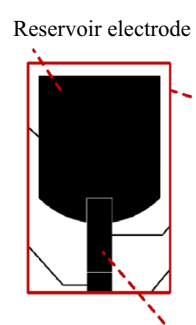

Dispensing electrode

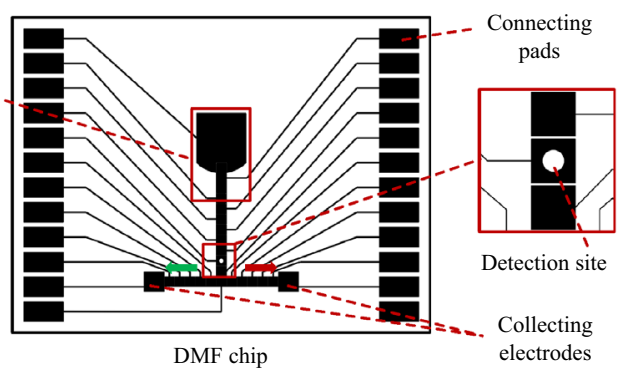

Fig. 1 The schematic of the DMF chip. The detailed dimensions of electrodes are as follows. The entire DMF chip is $4 \mathrm{~cm}$ long and $3 \mathrm{~cm}$ wide. The side length of reservoir electrode is $5 \mathrm{~mm}$. The length of the dispensing electrode is $3 \mathrm{~mm}$. The side length of the collecting electrodes is $2 \mathrm{~mm}$ and the side length of all other square electrodes is $1 \mathrm{~mm}$. The spacing between any two neighboring electrodes is $0.05 \mathrm{~mm}$. The diameter of the transparent circle is $0.6 \mathrm{~mm}$ in the detection site. The green arrow indicates the moving direction of fluorescent droplets while the red arrow represents the moving direction of non-fluorescent droplets. (Color figure online)
The fabrication process of the DMF chip is shown in Fig. 2. The glass substrate cleaned with acetone and isopropanol is first sputtered with a $200-\mathrm{nm}$ Al film. Then the Al film is patterned into a two-dimensional electrode array by standard lithography process and wet etching. Subsequently, a 3- $\mu \mathrm{m}$ Parylene layer is coated on top of the electrodes. Afterwards the Parylene surface is coated with a $100-\mathrm{nm}$ Teflon hydrophobic layer to complete the process of the bottom plate. An ITO glass coated with Teflon hydrophobic layer is used as the top plate and connected to the bottom plate by double-sided tapes.

\section{DMF platform and sorting principle}

Figure 3 shows the structure of the DMF droplet sorting platform. A comprehensive printed circuit board (PCB) is designed to modulate the input current signal and execute the task of driving voltage sequence from control circuit. The DMF chip is installed on the PCB through interface circuit. A fluorescence microscope equipped with a photoelectric multiplier tube (PMT) is utilized as fluorescence detection device. A signal generator and a high-voltage amplifier are employed as the power source to manipulate droplets. A control circuit is used to supply PCB with the electrode-driven voltage sequence which controls the generation, transportation and sorting of droplets.

When a droplet reaches the detection site, its fluorescence signal will be measured and compared with the signal collected right before the droplet's arrival. When an apparent (a)

(c)

(e)

(g)

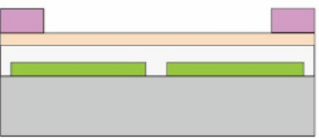

(b)

(d)

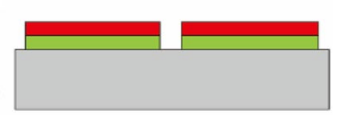

(f)

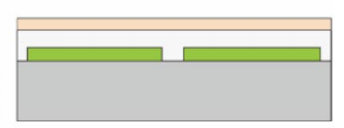

(h)

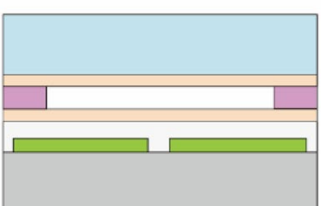

AZ5214 Parylene-C
Fig. 2 Schematic of chip fabrication process. a The substrate is sputtered with $\mathrm{Al}$ film. b-d The electrode array is formed by lithography process and wet etching. e-f The Parylene dielectric layer and Teflon hydrophobic layer are coated. $\mathbf{g}-\mathbf{h}$ The top plate is connected to the bottom plate by double-sided tapes 


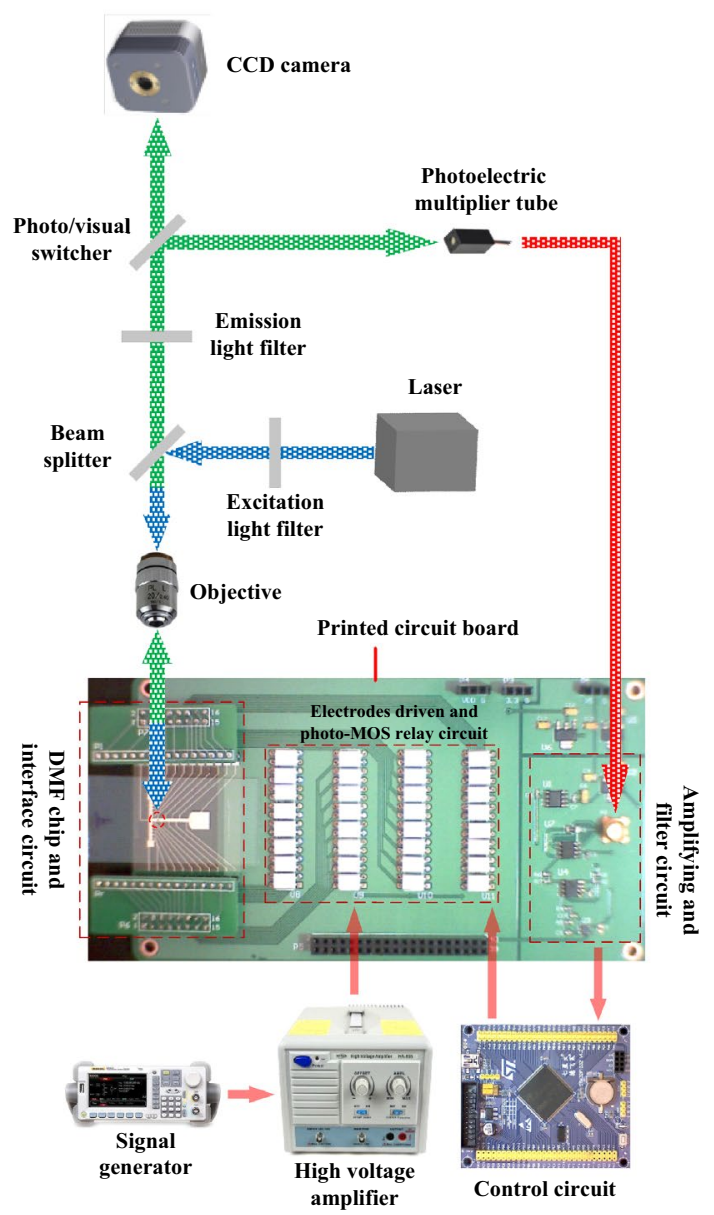

Fig. 3 Schematic structure of the DMF droplet sorting platform

increase of fluorescent signal is detected, the droplet is determined as fluorescent droplet and directed to the left collecting electrode on chip. On the contrary, if the fluorescence intensity decreases, the droplet will be determined as non-fluorescent droplet and directed to the right collecting electrode. Furthermore, the concentration of the fluorescent particles inside droplets can be conveniently quantified by the intensity of the fluorescent signal.

\section{Results and discussion}

\subsection{Droplet generation and driving}

Figure 4 shows the droplet dispensing and driving process of $10 \mu \mathrm{g} / \mathrm{mL}$ fluorescent particle solution. The driving power was $140 \mathrm{~V}, 50 \mathrm{~Hz} \mathrm{AC}$ and the driving rate of the droplet was $1 \mathrm{~Hz}$ in this experiment. Figure 4a shows the initial state of the large droplet on top of the reservoir electrode. In Fig. 4b, a liquid finger is stretched out of the large droplet by activating the dispensing electrode, then the liquid finger is pinched
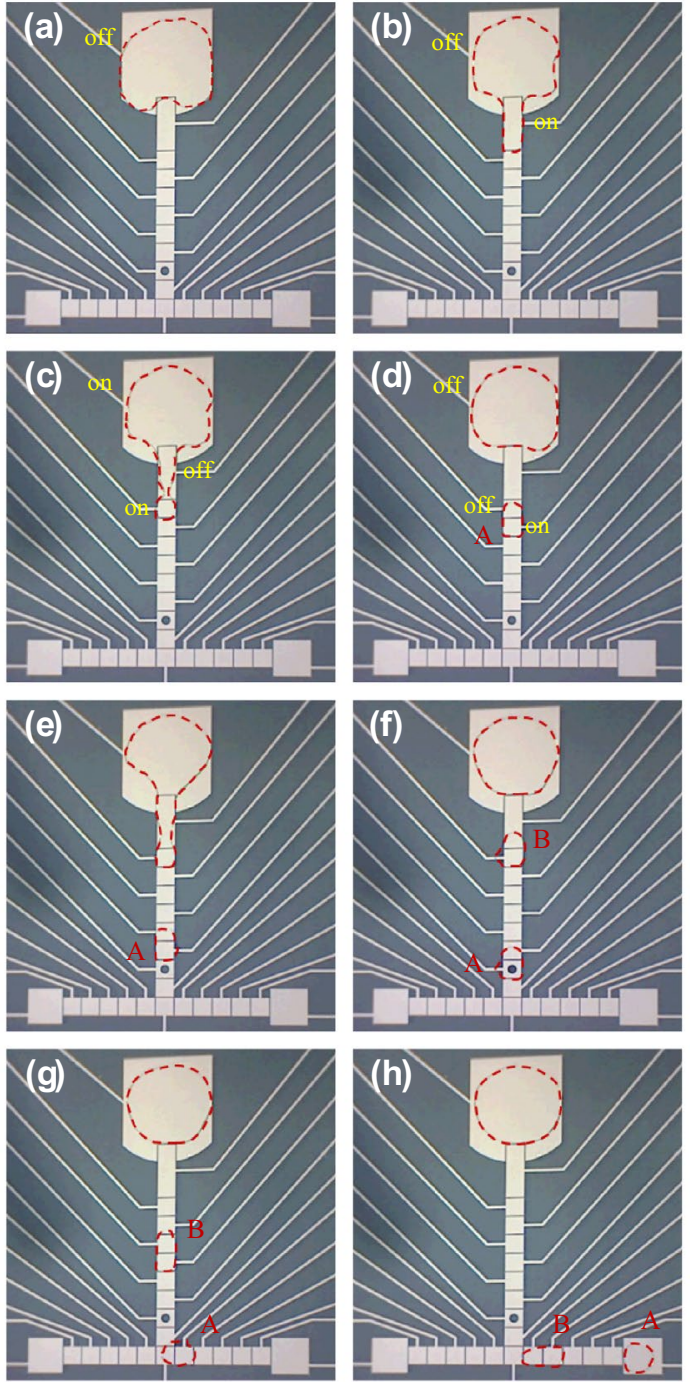

Fig. 4 The generation and driving process of $10 \mu \mathrm{g} / \mathrm{mL}$ fluorescent droplets. a-d The first droplet is dispensed. e-h The first droplet is detected and driven to the right collecting electrode meanwhile the second droplet is generated and detected

off in Fig. 4c to form the first small droplet A, and in Fig. 4d, the small droplet $\mathrm{A}$ is moved towards the detection site. When droplet A reaches the detection point, its fluorescent signal determines it as fluorescent droplet, and $\mathrm{A}$ is further moved to the right (Fig. $4 \mathrm{f}-\mathrm{h}$ ). Meanwhile, the second droplet $\mathrm{B}$ is dispensed, moved and detected according to the same sorting principle. In our tests, when the droplets driving rate exceeds $20 \mathrm{~Hz}$, the liquid finger cannot be pinched off in the process of (c), instead it returns to the reservoir electrode, in this case the droplet cannot be dispensed, so the highest droplets driving rate we obtained is $20 \mathrm{~Hz}$.

The volume of droplet A and droplet B is about $150 \mathrm{~nL}$. It has been observed that the dispensed drop volume is usually somewhat larger than the volume subtended by a 
square electrode; the reason is that a liquid tail formed after separation adds some additional liquid to the already formed droplet. It is this volume that causes the variability in the dispensing operation. In our previous study, we investigated major effects on droplet volume reproducibility. By proper parameter control, the droplet volume reproducibility is limited to $\pm 3 \%$ (Wang et al. 2011).

\subsection{Droplet measurement and sorting}

The droplet sorting process is tested with pure DI water solution, non-fluorescent particle solution $(100 \mu \mathrm{g} / \mathrm{mL})$, and fluorescent particle solution of varied concentrations $(1,10$,
100 , and $1000 \mu \mathrm{g} / \mathrm{mL}$ ), respectively. The driving voltage is $140 \mathrm{~V}, 50 \mathrm{~Hz} \mathrm{AC}$ and the driving rate of the droplet is $20 \mathrm{~Hz}$ in these experiments. The gain control voltage of PMT is $0.6 \mathrm{~V}$. Figure 5 shows the droplet sorting and fluorescence signal detection of pure DI water droplets and $100 \mu \mathrm{g} / \mathrm{mL}$ non-fluorescent particle droplets, respectively. From Fig. 5, it can be seen that the fluorescence intensity of non-fluorescent droplets is the same as that of pure DI water droplets. When the pure DI water droplet or non-fluorescent droplet passes the detection site, the signal drops by $6-8 \mathrm{mV}$. This result shows that the presence of non-fluorescent particles does not change the fluorescence signal detection; any blocking effect from non-fluorescent particles can be neglected.

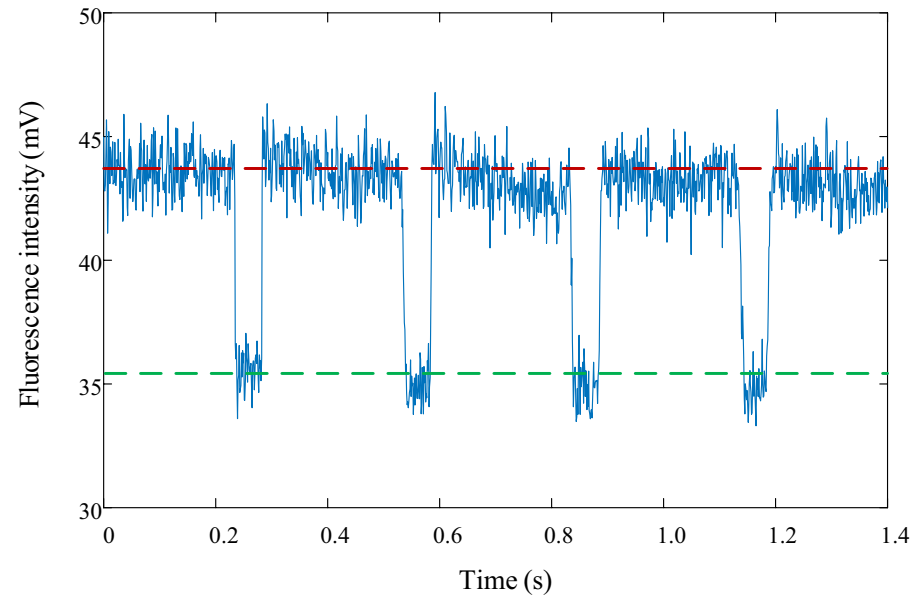

(a)

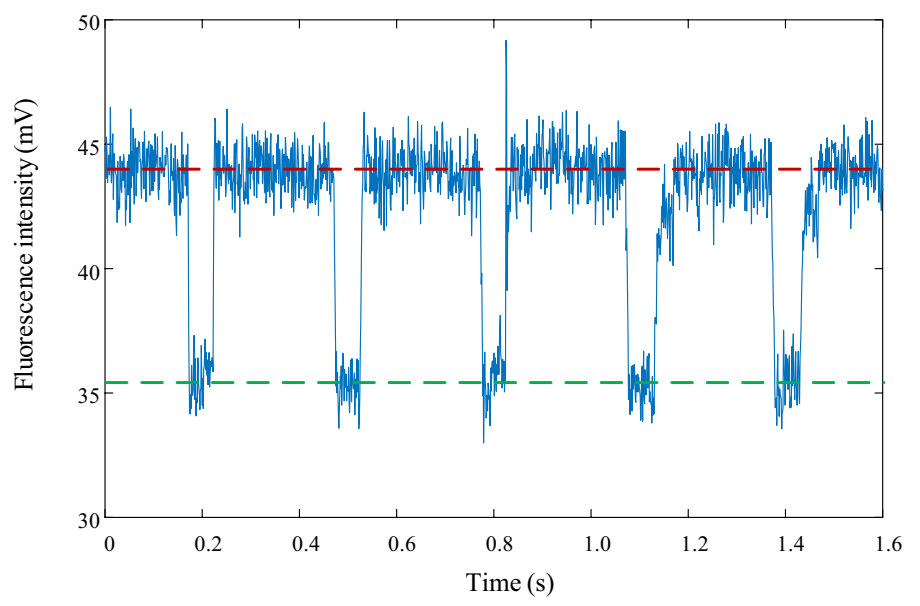

(c)

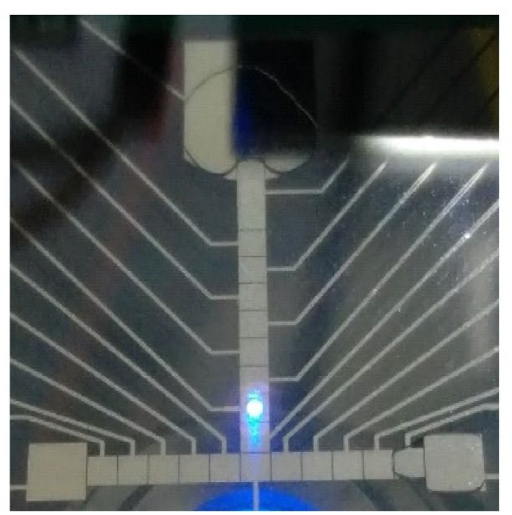

(b)

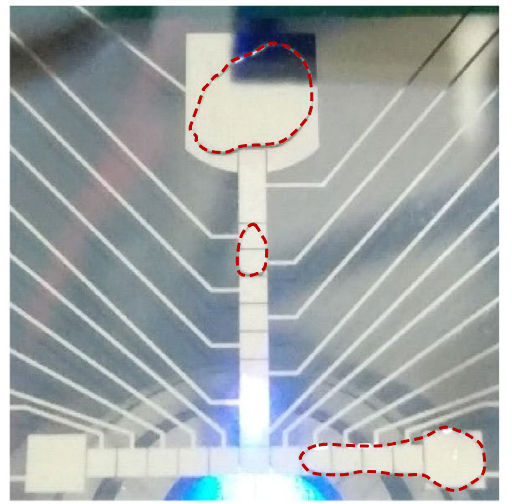

(d)
Fig. 5 a Fluorescence signal detection in the droplet sorting process of pure DI water solution. The red lines represent the fluorescence intensity when there is no droplet passing the detection site. The green lines denote the fluorescence intensity of pure ID water droplets. b Corresponding sorting result of pure DI water droplets. There are four pure ID water droplets collected to the right collecting electrode. c Fluorescence signal detection in the droplet sorting process of $100 \mu \mathrm{g} / \mathrm{mL}$ non-fluorescent particle solution. The red lines represent the fluorescence intensity when there is no droplet passing the detection site. The green lines denote the fluorescence intensity of non-fluorescent droplets. d Corresponding sorting result of non-fluorescent droplets. There are five non-fluorescent droplets collected to the right collecting electrode. (Color figure online) 
The actual droplet sorting is tested with fluorescent particle solutions. When a fluorescent particle solution is used, the dispensed droplet may contain certain fluorescent particles and also may contain even no fluorescent particle, especially when the particle concentration is low. Figure 6 shows the droplet sorting and fluorescence signal detection of droplets from $10 \mu \mathrm{g} / \mathrm{mL}$ fluorescent particle solution. Figure 6a depicts the change of signal intensity when six droplets pass the detection site. The existence of laser source and circuit noise causes the initial value of the detected fluorescence signal to be about $40 \mathrm{mV}( \pm 3 \mathrm{mV})$ when there is no droplet passing, while a signal peak will arise when a droplet arrives. The fourth signal peak is much higher than $40 \mathrm{mV}$ indicating that this droplet carries certain fluorescent particles, and according to the sorting principle, the droplet is collected to the left collecting electrode. The other five signal peaks are significantly lower than $40 \mathrm{mV}$ so these droplets carry no fluorescent particles and are collected to the right collecting electrode, as shown in Fig. $6 \mathrm{~b}$.

The number of fluorescent particles inside each droplet can be estimated using the Poisson distribution (Mazutis et al. 2013), in which the probability $P(X=x)$ of having $\mathrm{x}$ particles in one droplet is given by the equation $P(X=x)=\mathrm{e}^{-\lambda}\left(\lambda^{\mathrm{x}} / x !\right)$, where $\lambda$ is the mean number of fluorescent particles in each droplet. For example, when $\lambda$ is 0.3 , the dispensing process will result in $74.08 \%$ of the droplets containing no particles, $22.22 \%$ containing a single particle, $3.3 \%$ containing two particles and $0.38 \%$ containing more than two particles. According to the Poisson distribution, droplet occupancy can be conveniently tuned by changing the particle concentration or droplet volume.
To study the relationship between fluorescent intensity and the concentration of fluorescent particles, we tested four kinds of fluorescent particle solutions of $1,10,100$, and $1000 \mu \mathrm{g} /$ $\mathrm{mL}$. Figure 8 shows fluorescence images of droplets at each concentration. For each concentration, we dispense 100 droplets, and by allowing these droplets to pass through the detection site, we measure their fluorescent intensity. The average fluorescent intensity is shown in Fig. 7. A linear relationship is obtained between the fluorescent intensity and the particle concentration. Accordingly, the concentration of the fluorescent particles in any droplet can be conveniently determined using Fig. 7 as a standard reference curve. One thing that must be mentioned here is that the measured fluorescent intensity does not reflect all the fluorescent particles inside droplet because the size of the detection spot is smaller than the size of the droplet. However, given that the suspended particles

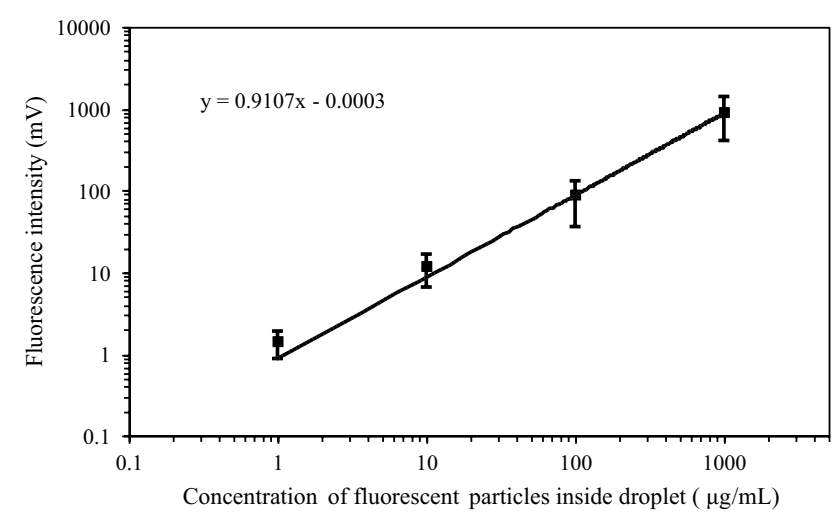

Fig. 7 The linear relationship between fluorescence intensity and the concentration of fluorescence particles inside droplet

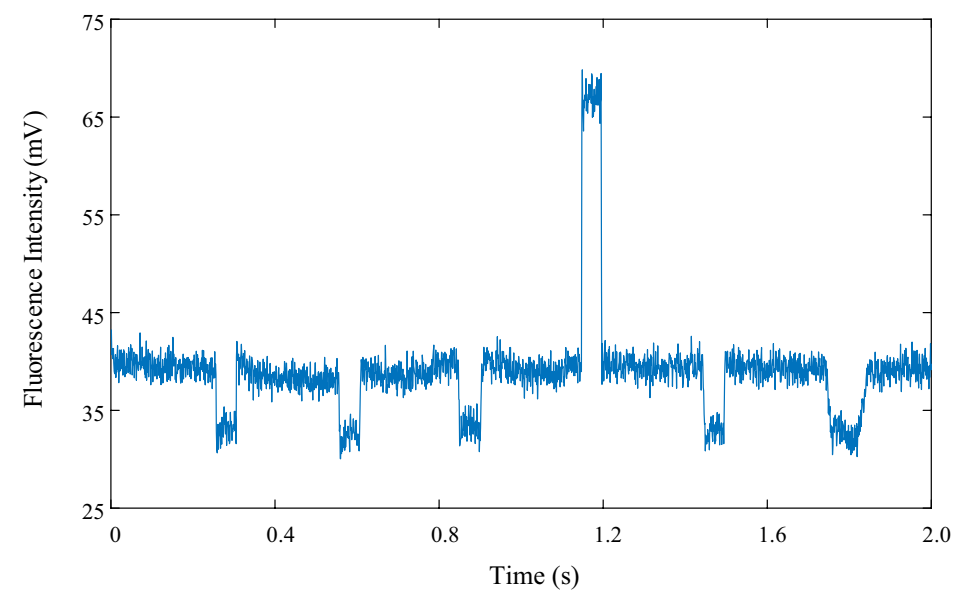

(a)

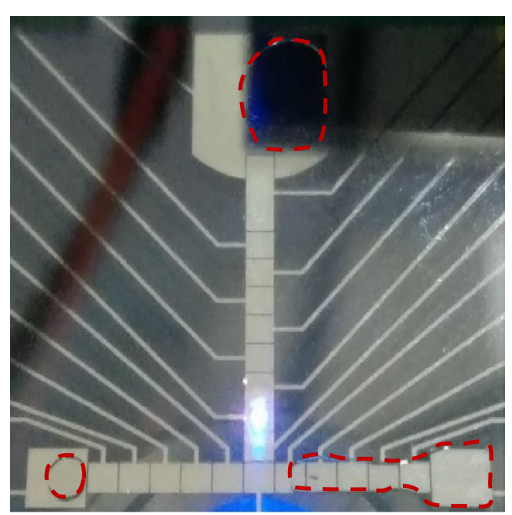

(b)
Fig. 6 a Fluorescence signal detection in the droplet sorting process of $10 \mu \mathrm{g} / \mathrm{mL}$ fluorescent particle solution. b Corresponding result of droplet sorting. There are five non-fluorescent droplets collected to the right collecting electrode and one fluorescent droplet collected to the left collecting electrode 

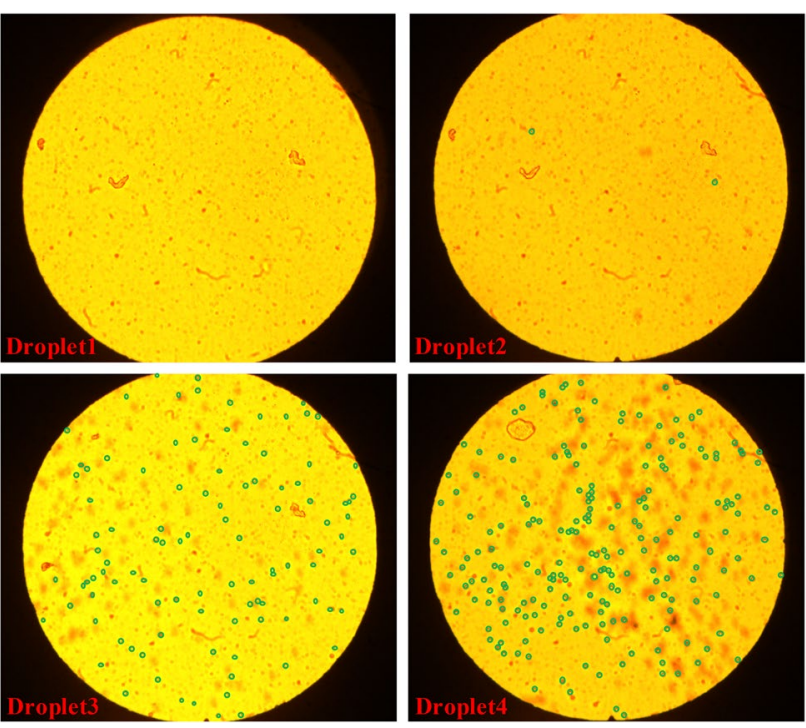

Fig. 8 Fluorescence images of fluorescent droplets. Droplet 1-Droplet 4 represent the fluorescent droplets of $1,10,100$, and $1000 \mu \mathrm{g} /$ $\mathrm{mL}$ fluorescent particle concentration, respectively. The green dots in images represent fluorescent particles. The average diameter of these fluorescent particles is $5 \mu \mathrm{m}$

distribute rather evenly inside the droplet, it is still valid to determine the number of fluorescent particles by the measured fluorescent intensity. A special case is that when there are only a few fluorescent particles inside each droplet, it is possible that these particles all stay outside the detection circle and cause detection failure. For a droplet containing $\mathrm{n}$ particles, the probability of detection failure can be calculated as $(1-R)^{n}$, here $R$ is the ratio of the area of the detection circle over the area covered by a droplet. In our experiment, $R$ is approximately 0.18 , so the failure probability approaches 0 when $\mathrm{n}$ is greater than 30 . As a result, the detection failure might occur when $n<30$, corresponding to a threshold particle concentration of $67.7 \mu \mathrm{g} / \mathrm{mL}$.

To avoid any failures when sorting low concentration fluorescent particle solutions, it is necessary to run multiple detections and use the average fluorescent intensity for quantification. But multiple detections are time consuming and limit further applications. A better solution is to use transparent ITO electrodes on the bottom substrate, thus all the fluorescent particles inside droplet can be detected. Further effort to develop this transparent scheme would be the focus of our future research.

\section{Conclusion}

In this paper, we design a kind of DMF chip and establish a DMF droplet sorting platform, on which we study the driving and sorting characteristics of nano-liter-sized liquid droplets encapsulating fluorescent-labeled particles. The droplets dispensed from on-chip reservoir are transported to a fluorescence detection site and sorted according to their fluorescence signals. The fluorescent droplets and non-fluorescent droplets are successfully separated and the number of fluorescent particles inside each droplet is quantified by its fluorescent intensity. We realize the sorting of droplets with a fluorescent particle concentration of $10 \mu \mathrm{g} / \mathrm{mL}$ at the driving rate of $20 \mathrm{~Hz}$. A linear relationship is also obtained between the fluorescent intensity and the particle concentration. Further work will focus on developing all transparent devices to solve the partial detection issue.

Acknowledgements This work was supported by National Science Foundation (no. 61504060), and by the Fundamental Research Funds for the Central Universities (30915118835, 30916011201, and 30915011302).

Open Access This article is distributed under the terms of the Creative Commons Attribution 4.0 International License (http://creativeco mmons.org/licenses/by/4.0/), which permits unrestricted use, distribution, and reproduction in any medium, provided you give appropriate credit to the original author(s) and the source, provide a link to the Creative Commons license, and indicate if changes were made.

\section{References}

Baret JC, Miller OJ, Taly V, Ryckelynck M, El-Harrak A, Frenz L, Rick C, Samuels ML, Hutchison JB, Agresti JJ, Link DR, Weitz DA, Griffiths AD (2009) Fluorescence-activated droplet sorting (fads): efficient microfluidic cell sorting based on enzymatic activity. Lab Chip 9(13):1850-1858

Cao Z, Chen F, Bao N, He H, Xu P, Jana S, Jung S, Lian H, Lu C (2012) Droplet sorting based on the number of encapsulated particles using a solenoid valve. Lab Chip 13(1):171-178

Chen Y, Wu TH, Kung YC, Teitell MA, Chiou PY (2013) 3D pulsed laser-triggered high-speed microfluidic fluorescence-activated cell sorter. Lab Chip 138(24):7308-7315

Clark IC, Thakur R, Abate AR (2018) Concentric electrodes improve microfluidic droplet sorting. Lab Chip 18(5):710-713

Eriksson E, Sott K, Lundqvist F, Sveningsson M, Scrimgeour J, Hanstorp D, Goksör M, Granéli A (2010) A microfluidic device for reversible environmental changes around single cells using optical tweezers for cell selection and positioning. Lab Chip 10(5):617

Hung S, Hsu CH, Chen C (2015) Cell sorting in microfluidic systems using dielectrophoresis. In: IEEE the 15th IEEE international conference on nanotechnology 2015, Rome, Italy. IEEE, pp 872-875

Hung PY, Jiang PS, Lee EF, Fan SK, Lu YW (2017) Genomic DNA extraction from whole blood using a digital microfluidic (DMF) platform with magnetic beads. Microsyst Technol 23(2):313-320

Johansson L, Nikolajeff F, Johansson S, Thorslund S (2009) On-chip fluorescence-activated cell sorting by an integrated miniaturized ultrasonic transducer. Anal Chem 81(13):5188-5196

Mazutis L, Gilbert J, Ung WL, Weitz DA, Griffiths AD, Heyman JA (2013) Single-cell analysis and sorting using droplet-based microfluidics. Nat Protoc 8(5):870-891

Obexer R, Pott M, Zeymer C, Griffiths AD, Hilvert D (2016) Efficient laboratory evolution of computationally designed enzymes with 
low starting activities using fluorescence-activated droplet sorting. Protein Eng Des Sel Peds 29(9):355

Okada H, Iguchi A, Iizuka R, Yoon DH, Sekiguchi T, Shoji S, Funatsu T (2014) Sensitive fluorescence-activated sorting of microdroplets containing subcellular structures by thermoreversible gelation polymer. In: 18th International conference on miniaturized systems for chemistry and life sciences, San Antonio, Texas, USA, MicroTAS 2014. Chemical and Biological Microsystems Society, pp 1259-1261

Qiao Y, Zhao X, Zhu J, Tu R, Dong L, Wang L, Dong Z, Wang Q, $\mathrm{Du}$ W (2017) Fluorescence-activated droplet sorting of lipolytic microorganisms using a compact optical system. Lab Chip 18(1): 190

Schmid L, Weitz DA, Franke T (2014) Sorting drops and cells with acoustics: acoustic microfluidic fluorescence-activated cell sorter. Lab Chip 14(19):3710-3718

Song H, Rosano JM, Wang Y, Garson CJ, Prabhakarpandian B, Pant K, Klarmann GJ, Perantoni A, Alvarez LM, Lai E (2015) Continuous-flow sorting of stem cells and differentiation products based on dielectrophoresis. Lab Chip 15(5):1320

Wang WQ, Jones T, Harding D (2011) On-chip double emulsion droplet assembly using electrowetting-on-dielectric and dielectrophoresis. Fusion Sci Technol 59(1):240-249
Wu L, Chen P, Dong Y, Feng X, Liu BF (2013) Encapsulation of single cells on a microfluidic device integrating droplet generation with fluorescence-activated droplet sorting. Biomed Microdevice 15(3):553-560

Xi HD, Zheng H, Guo W, Gañán-Calvo AM, Ai Y, Tsao CW, Zhou J, Li W, Huang Y, Nguyen NT, Tan SH (2017) Active droplet sorting in microfluidics: a review. Lab Chip 17(5):751

Zhao Y, Abdelfattah AS, Zhao Y, Ruangkittisakul A, Ballanyi K, Campbell RE, Harrison DJ (2014) Microfluidic cell sorter-aided directed evolution of a protein-based calcium ion indicator with an inverted fluorescent response. Integr Biol Quant Biosci Nano Macro 6(7):714-725

Publisher's Note Springer Nature remains neutral with regard to jurisdictional claims in published maps and institutional affiliations. 